\title{
ENXAQUECA COMPLICADA
}

\author{
A PROPÓSITO DE TRES CASOS
}

\author{
WILSON LUIZ SANVITO * \\ PAULO HELIO MONZILLO *
}

Ataques graves de enxaqueca podem se acompanhar de sinais e sintomas neurológicos, por vezes indistinguiveis de quadros vasculares tromboembólicos quer dos vasos do pescoço ou intracranianos 1,2,3,6,9. Fischer 6 refere que as manifestações neurológicas podem não ocorrer com as manifestações enxaquecosas habituais (quadro álgico) ou, até mesmo, sem antecedentes enxaquecosos. O conceito de enxaqueca complicada (EC) não é pacífico e sua caracterização clinica nem sempre é fácil. Pode ocorrer, nas fases iniciais do quadro, que a manifestação neurológica focal seja desacompanhada da fase álgica da enxaqueca. Nestas eventualidades, o dignóstico de enxaqueca pode ser postergado. No entanto, a presença de determinados sintomas neurológicos, mesmo desacompanhados de cefaléia, é altamente sugestiva de enxaqueca. O seguimento do paciente permite, na presença de manifestações oligo ou polissintomáticas, a caracterização da sindrome enxaquecosa. A conceituação de EC esbarra em vários tipos de dificuldade. Kalendovsky e Austin 10 referem que episódios de disfunção cerebral focal são observados em cerca de $1 / 3$ de pacientes com enxaqueca e que estes sintomas săo geralmente transitórios; às vezes as manifestaçōes focais determinam sequielas e podem até causar o óbito do paciente $4,5,7,10,11,12,13$. Serratrice adota a expressão enxaqueca associada ou acompanhada como sinônimo de enxaqueca complicada 16. Outras designações têm sido empregadas contribuindo para aumentar a confusão. Boisen cita as seguintes designações para a EC: "ataques enxaquecosos com lesão cerebral permanente"; "seqüelas permanentes no ataque da enxaqueca"; "enxaqueca focal"; "hemiplegia enxaquecosa" I Pearce refere que certas sequielas persistentes e não progressivas, após uma crise de enxaqueca - como os escotomas, as hemianopsias ou as hemiparesias -, são provavelmente resultado de um infarto isquêmico 15. Este autor inclui entre as enxaquecas complicadas, a enxaqueca oftalmoplégica. Para nossos propósitos adotamos critérios clínicos e/ou tomográficos na caracterizaçăo da $\mathrm{EC}$, além de um critério excludente em relação à enxaqueca oftalmoplégica. Do ponto de vista clínico, a manifestação cerebral focal deve determinar sequiela prolongada ou permanente, não sendo obrigatório a comprovação de lesăo cerebral estrutural ao exame tomográfico. Do ponto de vista tomográfico, é obrigatória a presença de infarto isquêmico

Trabalho da Disciplina de Neurologia do Departamento de Medicina da Santa Casa de Sāo Paulo: * Neurologista. 
após uma crise de enxaqueca. Neste caso, o diagnóstico de EC pode ser firmado mesmo na ausência de seqüelas neurológicas. Outro critério excludente é a presença de patologia estrutural subjacente como malformação vascular intracraniana, doença cerebrovascular oclusiva, hemorragia subaracnóidea, tumores intracranianos, traumatismos crânio-encefálicos e intoxicações exógenas. Também o uso de anticoncepcionais ou de medicação ergótica, em doses capazes de provocar distúrbios vasculares, deve ser considerado. Nestas eventualidades, até prova em contrário, o diagnóstico de EC não deve ser firmado. Em nossa opiniâo, a enxaqueca associada ou acompanhada não tem o mesmo significado de EC.

Os três casos, adiante relatados, preenchem os requisitos traçados nesta introdução.

\section{OBSERVAÇóES}

Caso 1 - A.C., branco, solteiro, comerciário, 32 anos de idade. Início há aproximadamente 10 anos com episódios de cefaléia holocraniana intensa e pulsatil, acompanhada de dificulāade visual e para se expressar. Foi atendido, na época. no Pronto Socorro da Santa Casa de São Paulo, ocasiăo em que se constatou disfasia mista e provável hemianopsia direita. Os exames complementares realizados naquela oportunidade (LCR, raios $\mathrm{X}$ de crânio, EEG e angiografia cerebral) nấo revelaram anormalidades. Uma semána após sua hospitalização, o quadro tinha regredido inteiramente. Desde essa época (1974) o paciente vem sendo acompanhado no ambulatório da Clinica Neurolúgica da Santa Casa e tem relatado episódios de escotomas negativos ou de defeito dı campo visual sob a forma de hemianopsia, seguidos de cefaléia pulsátil holocraniana ou sob a forma de hemicrania, acompanhada de náuseas $e$, às vezes, vômitos. A crise nem sempre se acompanha de cefaléia e frequentemente fica limitada aos distúrbios do campo visual. As vezes o quadro se acompanha de disfasia. Em 1979, uma crise de cefaléia e afasia mista se acompanhou de crise convulsiva do tipo GM. Nesta oportunidade foi submetido a tomografia axial computadorizada, que nada revelou de anormal. Voltou a apresentar convulsões em outras oportunidades, sendo medicado com fenobarbital. Dois eletrencefalogramas, realizados em 79 e 83, năo revelaram anormalidades. Nos últimos dias de fevereiro de 84 começou a sentir o hemicorpo direito estranho (mais pesado e diferente do lado esquerdo). Esta manifestação foi precedida de cefaléia pulsátil. Foi solicitada nova tomografia computadorizada em 5-4-84, cuja conclusāo fol: "Area de coeficiente de atenuacão diminuida na regiăo parietal posterior esquerda, apresentando efeito negativo de massa. Opinião: área de amolecimento na região descrita» (Fig. 1). Nesta época (inicio de abril), o paciente já estava assintomático. Durante este seguimento de 10 anos, o paciente foi submetido aos seguintes exames complementares: perfil lipídico, coagulograma, glicemia, ASLO, hemossedimentação, VDRL, hemograma, urina tipo I, LCR, EEG, raios $X$ de crânio, angiografia cerebral pelas carótidas e vertebrais te tomografia computadorizada. Todos os exames relacionados foram normais, com exceçăo da última tomografia já referida. 

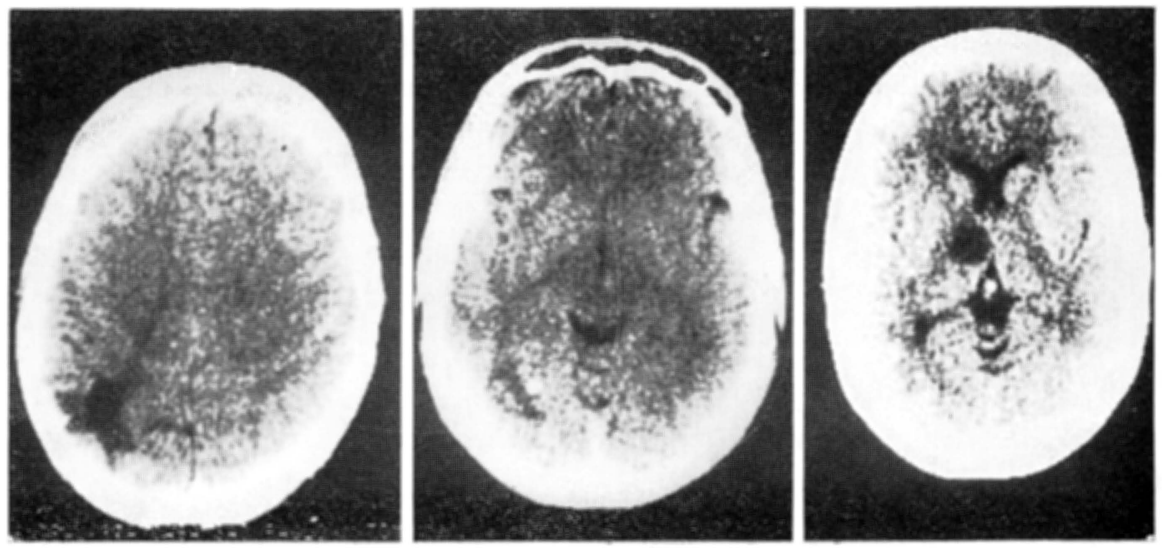

Fig. 1 - A esquerda, caso 1 (A.C.): area de coeficiente de atenuacão diminuida (hipodensa) na região parietal posterior esquerdia. No meio, caso ? (L.F.R.A.): área hipodensa na região occipital esquerda ao lado de calcificação nodular. $A$ direita. caso 3 (T.A.B.): área de coeficiente de atenuação diminuída (hipodensa) na vizinhança do tálamo optico esquerdo.

Cruso 2 - L.F.R.A., branco, solteiro, 25 anos, estudante de medicina. Início em 1972 (aos 14 anos de idade) com crise caracterizada por deficit visual do lado direito do campo, seguido de manjfestaçăo convulsiva. Na época foi internado na Beneficência Portuguêsa e acompanhado por neurologista que solicitou EEG (18-03-72) cuj.s traçado evidenciou: «sinais de disritnia paroxística temporal esquerda». A campimetria na época foi normal, assim como o exame do LCR. Passou a tomar Comital-L e permaneceu assintomático por 5 anos, então sofreu novo episódio traduzido por hemianopsia direita, acompanhada de mal-estar e vômito. Findo este episódio, passou bem mais alguns anos. Em 1982 apresentou quadro de cefaléia intensa (do tipo pulsátil) na região orbitária direita, acompanhada de hemianopsia e paresia do hemicorpo direito alguns minutos após. Na oportunidade o eletrencefalograna foi normal; também uma tomografia computadorizada nada revelou de anormal. Foi feito o diagnóstico de enxaqueca e medicado com Tegretal. Após este evento, apresentou vários episódios de defeito do campo visual do lado direito do campo, acompanhados de cefaléia de fraca intensidade. O último episódio foi em fins de fevereiro de 83 . Em 2-3-83 foi submetido à tomografia computadorizada, cuja conclusão foi: "Área hipodensa na regiāo occipital esquerda, sem efeito de massa, que após uso de contraste (dose dupla) não provocou acentuação da densidade. Presença de calcificașāo nodular adjacente à área hipodensa descrita. Opinião: área de isquemia cerebral na regiāo occipital esquerda, mais calcificacão nodular intraparenquimatosa» (Fig. 1). Foi feita em 22-8-84 tomografia de controle, na qual observa-se apenas a calcificação.

Caso 3 - T.A.B., branca, solteira, 29 anos de idade, médica. Em 5-6-82 apresentou quadro súbito de turvação visual e cefaléia, com duração aproximada de 1s horas. Refere que estava em seu domicilio, vendo televisão, quando começou a apresentar formigamento no lábio e língua do lado direito. Logo a seguir se instaloul ccfaléia 
fronto-parietal à esquerda, de fraca intensidade. Concomitantemente apresentou turvação visual, não conseguindo focalizar adequadamente a imagem do televisor. Uma vez instalada a turvaçāo visual, passou a sentir formigamento no membro superior direito, acompanhado de peso na måo direita e dificuldade na preensảo dos objetos. Também certo grau de desorientação e confusão se instalou, não conseguindo recordar-se de fatos. Por ocasiāo de sua admissão na santa Casa constatou-se déficit motor moderado braquiofacial à direita, abolição dos reflexos cutâneo-abdominais à direita e disfasia mista. o exame do aparelho cardiovascular näo revelava anormalidade. Tabagista de 5 cigarros por dia, nega etilismo e o uso de anticoncepcionais. $\mathrm{Na}$ oportunidade os seguintes exames complementares foram realizados: $\mathbf{L C R}$, raios $\mathrm{X}$ de crânio, raios $\mathrm{X}$ dos seios da face, carótido-angiografia esquerda, hemossedimentação, mucoproteinas, hemograma, eletroforese das proteinas séricas, ecocardiograma, EEG, ECG, campimetria e tomografia computadorizada. Todos os exames foram normais, com exceçāo da tomografia que revelou área de amolecimento isquêmfco na vizinhança do tálamo óptico esquerdo (Fig. 1). Após 7 dias, o quadro focal praticamente regrediu, permanecendo apenas abolição dos refłexos cutâneo-abdominais à direita. Em 3-2-83 refere episódio caracterizado por dificuldade para se expressar e para entender ordens, acompanhado de alteração visual (sensação de restrição concêntrica do campo visual) e cefaléia pulsátil. Em 4-5-84 refere episódio de redução do campo visual direito, seguido de adormecimento do membro superior direito e dificuldade para se expressar. Estas manifestações duraram aproximadamente 30 minutos e foram sucedidas por cefaléia moderada com duração de algumas horas.

\section{COMENTARIOS}

Nas enxaquecas acompanhadas pelo menos dois fatores têm sido implicados para justificar a ocorrência das manifestaçöes cerebrais focais $3,4,10,12$ : vasoconstriçăo, habitualmente presente na primeira fase da enxaqueca está diretamente relacionada com sua intensidade e duração; estado de hipercoagulabilidade sangüinea e hiperagregabilidade plaquetária, comprovadamente presentes em enxaquecosos. Além destes, outros fatores podem predispor ao aparecimento de infarto cerebral, como: evidências epidemiológicas da associação enxaqueca/hipertensão/doença cardiaca, cujo mecanismo permanece obscuro; o espasmo arterial, que ocorre na primeira fase da enxaqueca, leva a diminuição do fluxo sangüineo cerebral a niveis críticos para manter a vitalidade tecidual, sendo este efeito reforçado pela "inversão" do fluxo sangüíneo para as artérias extracranianas durante a fase dolorosa 5. Corroborando estes fatos, Dukes e Vieth em 1964 (citados por Hart e Miller ${ }^{8}$ ) encontraram diminuição progressiva do enchimento da artéria carótida interna durante a primeira fase da enxaqueca, contrastando com angiogramas normais durante a fase álgica. Estes achados coincidem com as medidas do fluxo sangüineo cerebral, realizadas por Skinh $\varnothing \mathbf{j}$ em 1971 (citado por Boisen 1). O elenco de eventos presente na crise enxaquecosa, seja de natureza vascular (espasmo arterial, edema da parede do vaso) seja de natureza sangüinea (hipercoagulabilidade sangüínea, hiperagregabilidade plaquetária, alentecimento do fluxo) pode justificar quadros vasculares em pacientes jovens sem vasculopatias 10. Para alguns autores 1,10, outro fator potencializador de quadros tromboembólicos seria o uso prolongado de estrógenos em enxaquecosas. Este fato estaria diretamente envolvido com 0 
aumento dos fatores de coagulação, bem como da atividade plaquetária, além de provocar "endurecimento" da parede vascular, favorecendo as possibilidades de coagulação local. Os enxaquecosos também apresentam aumento da concentração de ácidos graxos livres antes e/ou durante a crise, assim como redução de sua capacidade para metabolizá-los 10. Alguns fatos corroboram a tese da possibilidade de ocorrência de isquemia cerebral durante uma crise enxaquecosa. Furthermore e Barolin (citados por Boisen 1) demonstraram alterações metabólicas de produção de energia no parênquima cerebral, assemelhadas às encontradas na encefalomalácia. Também alterações liquóricas como lactoacidose, aumento da GABA e AMP cíclico confirmam a hipótese de isquemia cerebral 2,11.

Os quadros de EC podem ser classificados de acordo com a duração das manifestaçōes neurológicas focais, em: (1) transitórios (duração inferior a 24 horas); (2) prolongados (1 dia a 3 meses); (3) permanentes. $O$ nosso caso 2 se enquadra no item 1 desta classificação, enquanto os casos 1 e 3 se enquadram no item 2. Bousser e col.2 traçam alguns critérios para a caracterização da EC: "As crises devem ocorrer de maneira recorrente com inicio antes dos 30 anos de idade, devendo o quadro ser encarado de maneira diferente quando tem início depois dos 40 anos". Neste caso o diagnóstico diferencial com doença cerebrovascular se impõe. Já Kalendovsky e Austin 10 classificam os pacientes em dois grupos: (1) aqueles com crises enxaquecosas desde jovem e com evolução geralmente benigna; (2) aqueles com crises graves, incidindo numa fase mais tardia da vida, podendo no decurso de uma crise ocorrer infarto cerebral. Os nossos casos estão enquadrados nos critérios de Bousser e col., uma vez que o inicio da sintomatologia recorrente ocorreu antes dos 30 anos. As manifestaçóes neurológicas focais dos enxaquecosos podem ocorrer desacompanhadas da fase álgica. A expressão clínica da sintomatologia é variada: turvação visual, escotomas, hemianopsias, déficits motores lateralizados, déficits sensitivos, distúrbios da linguagem e, por vezes, sintomas sugerindo comprometimento do tronco cerebral. Cerca de $2 / 3$ dos enxaquecosos apresentam sintomatologia focal e em aproximadamente $1 / 3$ a sintomatologia é visual. Deve-se ressaitar que dois de nossos pacientes, além da sintomatologia enxaquecosa focal, apresentaram também crises convulsivas do tipo GM.

O diagnóstico de EC poderá ser firmado através de critérios clínicos e/ou neurorradiológicos. Angiografias cerebrais têm sido realizadas em enxaquecosos e, excluindo-se os casos de malformação vascular, estes exames têm-se revelado normais na maior parte dos casos. Embora a oclusāo de vasos intracranianos seja excepcional ao exame angiográfico nos enxaquecosos, já foram relatados pelo menos três casos com oclusão da artéria cerebral posterior, um caso de oclusão da cerebral média e um caso da cerebral anterjor 2,3,5. Entretanto o exame eletivo, nestes casos, é a tomografia computadorizada que pode evidenciar áreas de infarto, mesmo na ausência de alteraçōes angiográficas. Das alterações tomográficas, três seriam as principais $8,10,12,13$ : (1) áreas hipodensas no parênquima cerebral; (2) dilatação ventricular; (3) atrofia cortical. As áreas hipodensas significam infarto isquêmico, embora o desaparecimento relativamente rápido da área, nos exames subseqüentes, possa sugerir apenas edema cerebral. Geralmente estas lesões hipodensas não apresentam efeito de 
massa, salvo quando vêm acompanhadas de edema acentuado 11. Estes dados coincidem com os da nossa pequena casuística.

São escassas as informações sobre os efeitos tardios de crises enxaquecosas graves e repetidas. Sabe-se, porém, que insultos isquêmicos recorrentes podem determinar alterações cerebrais como atrofia e dilatação ventricular 11 . A responsabilidade da enxaqueca em atrofias cerebrais generalizadas é altamente questionável, entretanto o significado de uma atrofia localizada é diferente, já que representa quase sempre reliquat de infarto 9 . Deve ser ressaltado ainda que a presença de tomografia normal após a crise não exclui a possibilidade de lesão isquêmica, devendo-se repetir o exame em fases mais tardias. Por vezes, a lesão só se torna aparente após a infusão de grande volume de contraste 5 .

\section{RESUMO}

Três casos de enxaqueca complicada são relatados, de acordo com critérios previamente estabelecidos. A expressão enxaqueca complicada deve ser reservada para os casos em que sintomas neurológicos focais ultrapassam a crise enxaquecosa por mais de 24 horas ou quando déficit neurológico permanente se desenvolve em virtude de infarto cerebral. Outro critério para firmar o diagnóstico é a presença de infarto na tomografia computadorizada, mesmo na ausência dos critérios clínicos mencionados. $O$ papel da agregaçăo plaquetária, do espasmo vascular e da hipercoagulabilidade do sangue é considerado na causa do infarto. Todos os doentes, do presente relato, apresentaram déficit neurológico prolongado e infarto ao exame tomográfico.

\section{SUMMARY}

\section{Complicated migraine: report of three cases.}

After brief considerations about complicated migraine, three cases are reported. The diagnosis was made by the clinical features and by computed tomography. The term complicated migraine must be reserved for those cases in which the neurological symptoms and signs outlast migraine attack for more than 24 hours or in which permanent deficit develops because of cerebral or brainstem infarction. Other criteria for diagnosis is the presence of infarction in CT scanning, although prolonged or permanent deficit are absent. The possible role of platelet aggregation, vascular spasm and increased coagulability of the blood, as the cause of infarction, is discussed. All the patients in this report showed prolonged neurological deficit and infarction in CT scanning.

\section{REFERENCIAS}

1. BOISEN, E. - Strokes in migraine: report on seven strokes associated with severe migraine attacks. Dan. med. Bull. 22:100, 1975.

2. BOUSSER, M.G.; BARON, J.C.; IBA-ZIZEN, M.T.; COMAR, D.; CANABIS, E. \& CASTAIGNE, P. - Migrainous cerebral infarction: a tomographic study of cerebral blood flow and oxygen extraction fraction with the oxygen-15 inhalation tecnique. Stroke 11:145, 1980, 
3. CASTALDO, J.E.; ANDERSON, M. \& REEVES, A.G. - Middle cerebral artery occlusion with migraine. Stroke 13:308, 1982.

4. COHEN, R.J. \& TAYLOR, J.R. - Persistent neurologic sequelae of migraine: a case report. Neurology 29:1175, 1979.

5. DORFMAN, L.J.; MARSHALL, W.H. \& ENZMANN, D.R. - Cerebral infarction and migraine: clinical and radiologic correlations. Neurology 29:317, 1979.

6. FISCHER, C.M. - Late-life migraine accomppaniments as a cause of unexplained transient ischemic attacks. J. Can. S. Neurol. 7:9, 1980.

7. GUEST, I.A. \& WOLF, A.L. - Fatal infarction of brain in migraine. Brit. med. J. $1: 225,1964$.

8. HART, R.G. \& MILLER, V.T. - Cerebral infarction in young adults: a practical approach. Stroke 14:110, 1982.

9. HUNGERFORD, G.D.; BOULAY, G.H. \& ZILKHA, K.J. - Computorized axial tomography in patients with severe migraine: a preliminary report. J. Neurol. Neurosurg. Psychiat. 39:990, 1976.

10. KALENDOVSKY, Z. \& AUSTIN, J.H. - "Complicated migraine" its association with increased platelet aggregability and abnormal plasma coagulation factors. Headache $4: 18,1975$.

11. MATHEN, N.T.; MEYER, J.S.; WELCH, K.M.A. \& NEBLETT, C.R. - Abnormal CT-scans in migraine. Headache 16:272, 1977.

12. MCDONALD, W.I. \& SANDERS, M.D. - Migraine complicated by ischemic pappillopathy. Lancet $4: 521,1971$.

13. MOOREHEAD II, M.T.; MOVIUS, H.J.; MOOREHEAD, J.R.; JACKSON, M.H. \& JACKSON, J.G. - Classic migraine with cerebral cortical infarction causing permanent hemianopsia. Southern med. J. 2:821, 1979.

14. OSUNTOKUN, B.O. \& OSUNTOKUN, O. - Coomplicated migraine and haemoglobin AS in Nigerians. Brit. med. J. 2:621, 1972.

15. PEARCE, J. - Migraña complicada. In J. Pearce: Conceptos Actuales en la Migraña. Marin, Barcelona, 1977, pg. 39.

16. SERRATRICE, G. - Saber Interpretar uma Enxaqueca. Andrei, São Paulo, 1982.

Disciplina de Neurologia, Departamento de Medicina da Santa Casa - Rua Dr. Cesário Motta, Jr., 112 - 01221 - Säo Paulo, SP - Brasil. 